\title{
Facial Recognition in Collaborative Learning Videos ${ }^{\star}$
}

\author{
Phuong $\operatorname{Tran}^{1}$, Marios Pattichis ${ }^{1}$, Sylvia Celedón-Pattichis ${ }^{2}$, and Carlos LópezLeiva ${ }^{2}$ \\ 1 Dept. of Electrical and Computer Engineering \\ University of New Mexico, Albuquerque NM 87106, USA \\ 2 Dept. of Language, Literacy, and Sociocultural Studies \\ University of New Mexico, United States.
}

\begin{abstract}
Face recognition in collaborative learning videos presents many challenges. In collaborative learning videos, students sit around a typical table at different positions to the recording camera, come and go, move around, get partially or fully occluded. Furthermore, the videos tend to be very long, requiring the development of fast and accurate methods.

We develop a dynamic system of recognizing participants in collaborative learning systems. We address occlusion and recognition failures by using past information about the face detection history. We address the need for detecting faces from different poses and the need for speed by associating each participant with a collection of prototype faces computed through sampling or K-means clustering. Our results show that the proposed system is proven to be very fast and accurate. We also compare our system against a baseline system that uses InsightFace [2] and the original training video segments. We achieved an average accuracy of $86.2 \%$ compared to $70.8 \%$ for the baseline system. On average, our recognition rate was 28.1 times faster than the baseline system.
\end{abstract}

Keywords: Human front-face detection and recognition · video analysis.

\section{Introduction}

We study the problem of face recognition in collaborative learning environments. Our goal is to develop fast and accurate methods that can be used to quantify student participation as measured by their presence in their learning groups.

Fig. 1 represents an example of a collaborative learning group. A collaborative learning group is represented by the group of students closest to the camera. Background groups are not considered part of the collaborative group that we are analyzing. There is a possibility that students or facilitators move between groups. Thus, we need to recognize the current members of our group from a larger group of students.

A fundamental challenge of our dataset is that face recognition needs to work at a large variety of poses. As long as a sufficiently small part of the face is visible, we need to identify the student. As an example, in Fig. 1, the student on the front right with a white hoodie has his face mostly blocked. Furthermore, students may disappear or

\footnotetext{
* This material is based upon work supported by the National Science Foundation under Grant No.1613637, No.1842220, and No.1949230.
} 


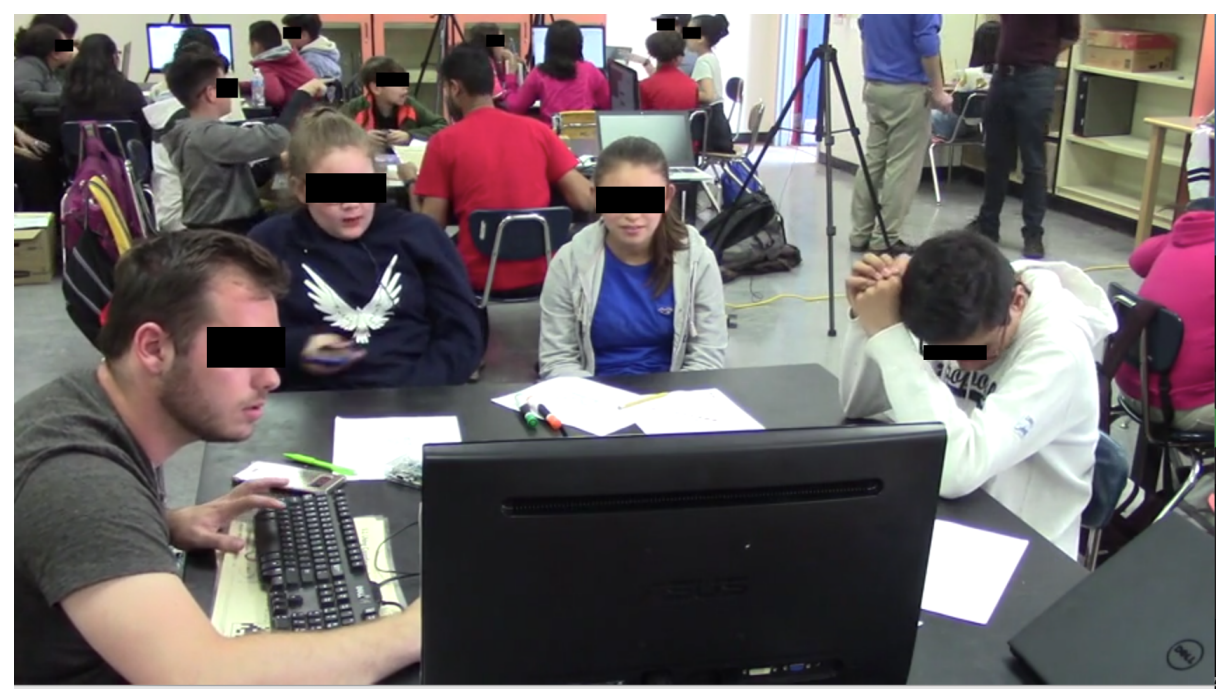

Fig. 1. Example of a collaborative learning group. We seek to recognize the students who are closer to the camera while ignoring students from all other groups.

reappear because the camera moves, or the students take a break, or because they have to leave the group. Hence, there are significant video properties to our problem that are not present in standard face recognition systems.

As part of a collaborative project between engineering and education, our goal is to assist educational researchers in analyzing how students who join the program learn and facilitate the learning of other students. The problem of identifying who is crucial for assessing student participation in the project. Furthermore, the developed methods need to be fast. Eventually, we will need to apply our methods to approximately one thousand hours of videos that we need to analyze.

The standard datasets for face recognition use a database of front-facing images. The Labeled Faces in the Wild (LFW) dataset [3] contains more than 13,000 face images with various poses, ages, and expressions. The Youtube Face (YTF) dataset [5] contains around 3,500 videos with an average range of 181 frames/video from 1,600 different people. The InsightFace system [1] developed the use of Additive Angular Margin Loss for Deep Face Recognition (ArcFace) on a large-scale image database with trillions of pairs and a large-scale video dataset, and tested on multiple datasets with different loss function models (ArcFace, Softmax, CosFace,..). InsightFace gave the best accuracies on LFW and YTF with $99.83 \%$ 98.02\%. We adopt InsightFace as our baseline face recognition system because of their state-of-the-art performance.

We also provide a summary of video analysis methods that were developed specifically by our group for analyzing collaborative learning videos.

In [4], the authors introduced methods for detecting writing, typing, and talking activities using motion vectors and deep learning. In [6], the authors developed methods to detect where participants were looking at. In [7], the authors demonstrated that FM 
images with low-complexity neural networks can provide face detection results that can only be achieved with much more complex deep learning systems.

We provide a summary of the contributions of the current paper. First, we introduce the use of a collection of face prototypes for recognizing faces from different angles. Second, we apply multi-objective optimization to study the inter-dependency between recognition rate, the number of face prototypes, and recognition accuracy. Along the Pareto front of optimal combinations, we select an optimal number of face prototypes that provides for a fast approach without sacrificing recognition accuracy. Third, we use the past recognition history to deal with occlusions and, hence, support consistent recognition throughout the video. Compared to InsightFace [2], the proposed system provides for significantly faster recognition rates and higher accuracy.

We summarize the rest of the paper into three additional sections. In section 2 we provide a summary of our methodology. We then provide results in section 3 and concluding remarks in section 4

\section{Methods}

We present a block diagram of the overall system in Fig. 2 Our video recognition system requires a set of face prototypes associated with each participant. The video face recognition algorithm detects the faces in the input video and computes minimum distances to the face prototypes to identify each participant. To handle occlusion issues, the system uses past detection history.

\subsection{Computation of Face Prototypes}

We use two different methods to compute the face prototypes. First, we consider the use of K-means clustering to select a limited number of face prototypes from the training videos. Second, we consider a baseline approach where we use sparse sampling of the training videos to define the face prototypes. To achieve sparsity, we use one sample image per second of video. For our second approach, we used a video from Group D from urban cohort 2 level 1 (C2L1, Video 4) and Group E from C3L1 (Video 5), whereas the first approach tested on Group C from urban C1L1 (Video 1). Each sampled face is aligned and resized to $112 \times 112$.

We summarize the K-means approach in Fig. 2. We use K-means to cluster similar frames that appear when a student does not move very much. Hence, we expect that the centroids that result from the K-means algorithms would represent a small number of diverse face poses for each participant. To avoid unrealistic centroid images, we compute our face prototypes using the images closest to our cluster centroids. After finding a prototype image that is closest to the mean, we align and resize each prototype image to $112 \times 112$.

We use multi-objective optimization to determine the minimum number of face prototypes without sacrificing classification accuracy. We present our results for a representative video from group C, level 1, in Fig. 3 . For the optimization, we use a logbased search by varying the number of face prototypes from $2^{0}$ to $2^{11}$. For K-means clustering, we see that the accuracy peaks at $79.8 \%$ for 1024 face prototypes, with a 


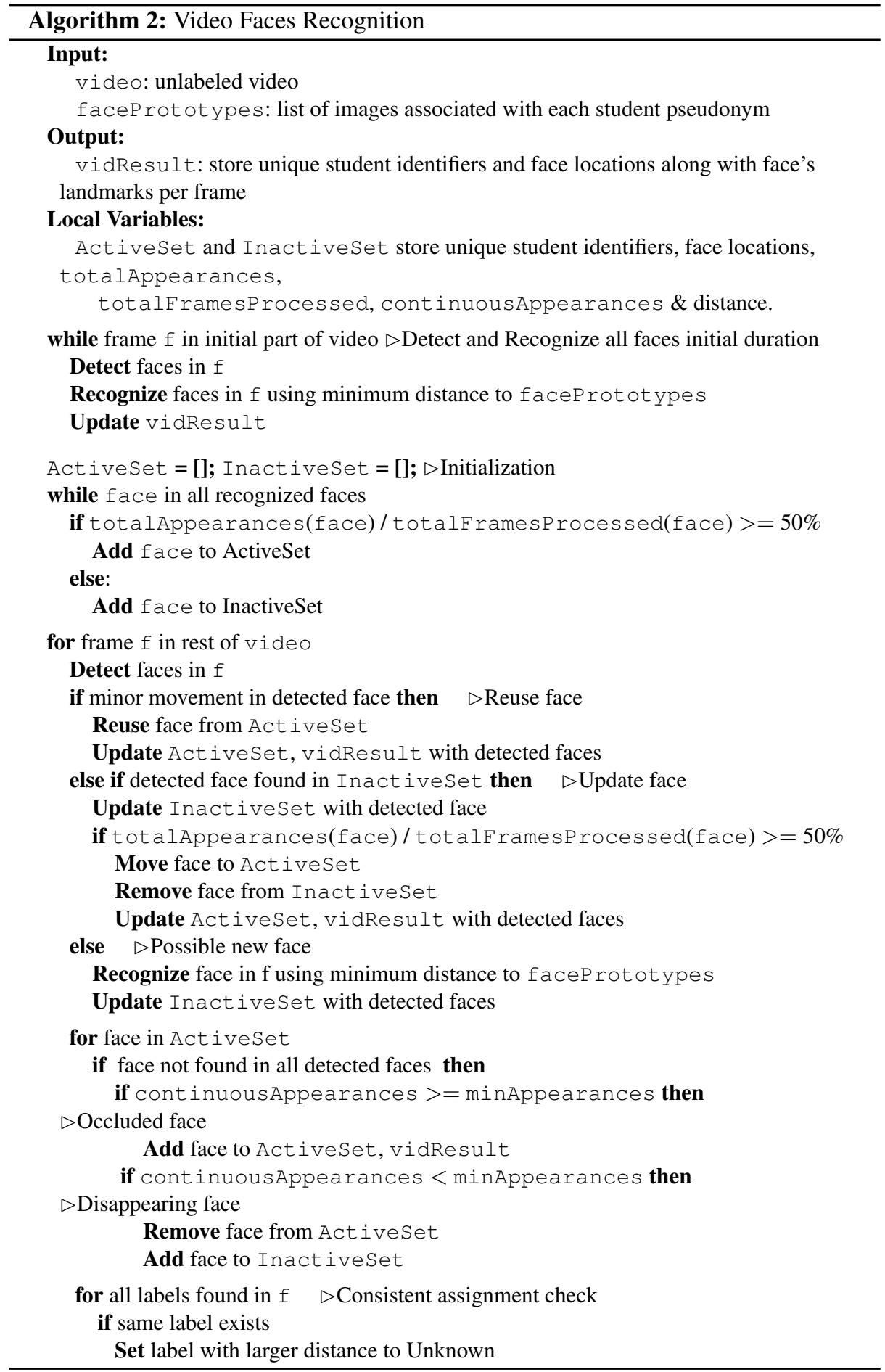



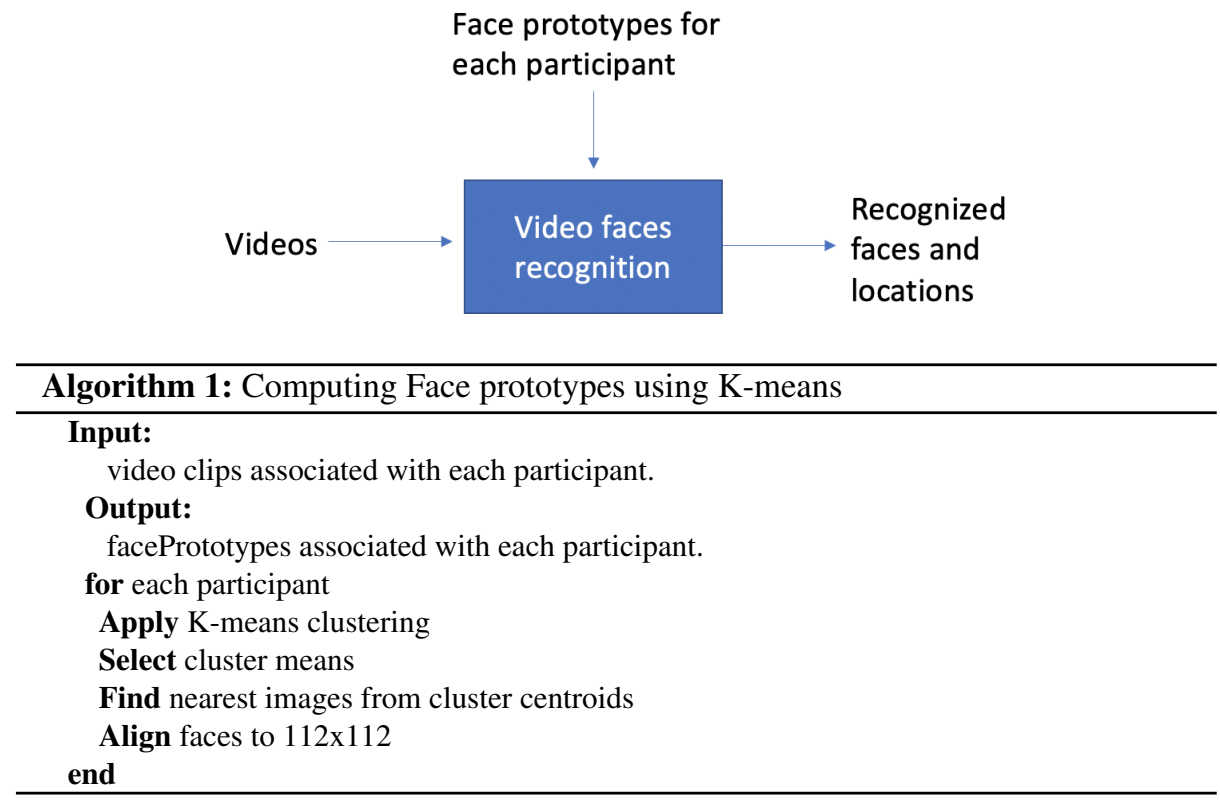

Fig. 2. Block diagram for recognizing faces from videos. Each face is associated with a collection of face prototypes. The method computed face prototypes using video sampling or K-means clustering. K-means clustering is given here.

recognition rate of 4.8 seconds per frame. In this example, we only used one video to validate our approach. We expand the validation set to more videos as described in [8]. This process run on a personal Macbook Pro that ran Mac-OS with 2.3GHz, 4-core, Intel i5 processors.

\subsection{Video Faces Recognizer}

We present the algorithm for video face recognition in Algorithm 2. The input is an unlabeled video and the faceprototypes that provides a list of images associated with each participant. The detected faces for each video frame are returned in vidResult. To address occlusion, the algorithm maintains the face recognition history in Activeset and InactiveSet.

First, for the first two seconds of the videos, we detect all participants in each video frame using MTCNN [9]. For each detected face, MTCNN computes five landmark points: two for the eyes, one for the nose, and two for the mouth corners. The face detector uses a minimum area requirement to reject faces that belong to another group. Thus, we reject smaller face detections that are part of another group or other false positives because they appear smaller in the video. Second, we classify each detected face by selecting the participant that gives minimum distance to their associated prototypes stored in facePrototypes. 
We use the initial face recognition results to initialize Activeset and Inactiveset. The faces that have been recognized in more than half of the frames are stored in the ActiveSet. The rest of them are stored in the Inactiveset. For each face detection, we use a dictionary to store: a pseudonym, location information, totalAppearances that stores the total number of frames where this face was detected, and totalFramesProcessed which represents the total number of frames processed since this face appeared for the first time. Hence, we use the Activeset to hold faces that appear consistently whereas Inactiveset contains faces that are still in doubt.

When a recognized face enters the Activeset, it gets a maximum value of 10 for its corresponding continuousAppearances. When a previously recognized face is missing, continuousAppearances gets decremented by 1 . When a face re-appears, continuousAppearances is incremented until it reaches 10 again. We also set minAppearances to 5 as the minimum requirement on the number of prior continuous appearances for addressing occlusion issues. Thus, for each face in the Activeset that is not being detected in any frame, if continuousAppearances $\geq$ minAppearances, we declare the face as occluded, we mark it as present, and update vidResult. Else, if continuousAppearances < minAppearances, we declare the face as disappearing, and move it to the Inactiveset.

We thus process the rest of the video based on the following four cases:

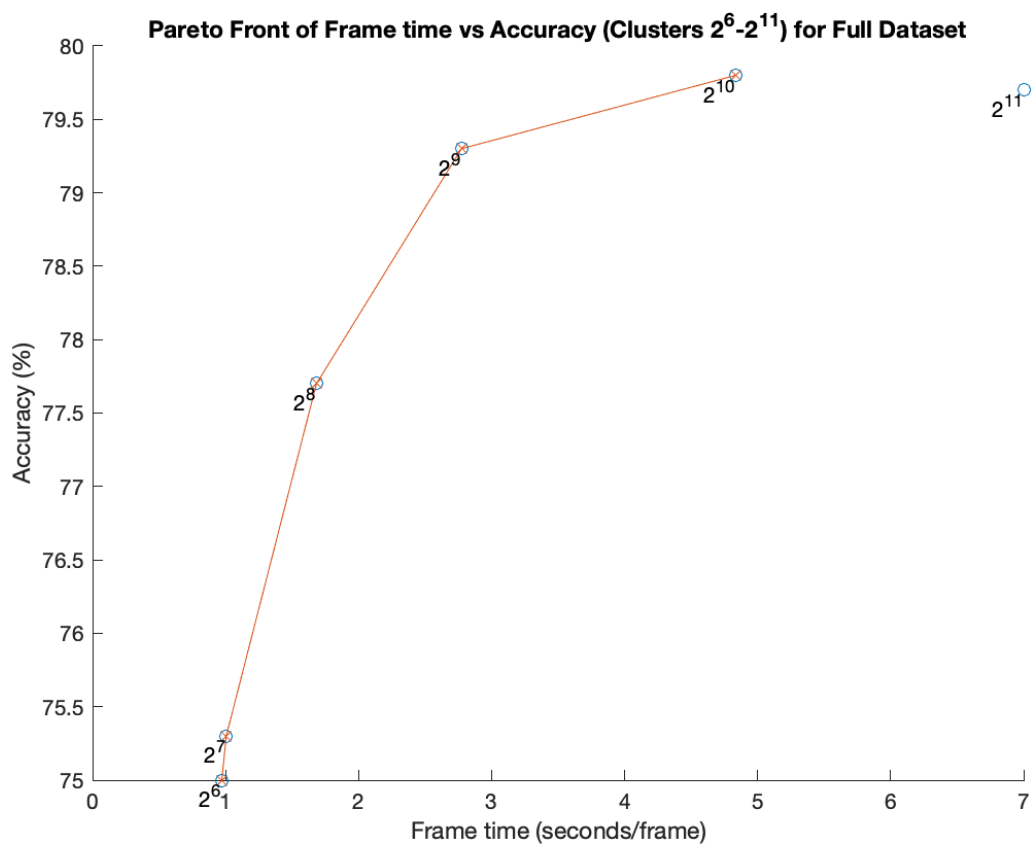

Fig. 3. Pareto Front on the K-mean Clustering Results 
(i) If a newly detected face corresponds to a minor movement of a prior detection, we keep it in the ActiveSet. This approach leads to a significant speedup in our face recognition speed.

(ii) If a newly detected face is in the Inactiveset, we update the Inactiveset with the new detection, and look at the ratio of totalAppearances/totalFramesProcessed to determine if it needs to move to the ActiveSet. Otherwise, the face stays in Inactiveset.

(iii) If a newly detected face does not belong to either set, then recognize it and move it to the Activeset.

(iv) If a face that belongs to the ActiveSet no longer appears, we consider the case for occlusion and that the participant has left the frame. As described earlier, we check cont inuousAppearances to determine whether to declare the face occluded or not.

Lastly, we do not allow the assignment of the same label to two different faces in the same frame. In this case, the face that gives the minimum distance is declared the recognized face while the other(s) are declared Unknown.

\section{Results}

We sampled twenty-four participants from our video dataset (11 boys and 13 girls). For training, we used $80 \%$ of the data for fitting and $20 \%$ for validation using different video sessions. From our video sessions, we randomly select short video clips of 5 to 10 seconds for training our video face recognizer. Overall, we use more than 200,000 video frames from 21 different video sessions. We have an average of about 10,000 image examples per participant. Furthermore, as the program lasted three years, the testing dataset used later on videos (e.g., later cohorts and different levels). For the testing dataset, we used seven video clips with a duration of 10 to 60 seconds. However, we do assume that we have trained for all of the students within the collaborative group. For reporting execution times, we use a Linux system with an Intel(R) Xeon(R) Gold 5118 CPU running @ 2.30GHz with 16GB Memory and Nvidia Quadro RTX 5000 GPU with 3072 Cuda cores.

We present face recognition accuracy results in Table 1 using said system. For InsightFace, we use all of the training video frames as face prototypes. From the results, it is clear that the proposed method is far more accurate than the baseline method. The difference in accuracy ranged from as low as $2 \%$ to as large as $25 \%$. Out of 19 participants in these five videos, our method achieved higher or same accuracy in 17 cases. Overall, our method achieved $86.2 \%$ compared to $70.8 \%$ for the baseline method.

We present face recognition examples in Fig. 4 Figs. 4 (a) and 4 (b) show results from the same video frame of Video 2. The baseline method recognized Javier67P (lower right in (a)) and Kenneth1P (white shirt with glasses in (a)) as Unknown, whereas our approach correctly identified all four participants. A second example for video 5 is presented in Figs. 4(b) and (e). The baseline method identified all three people correctly. However, the baseline method also detected and incorrectly claimed recognition of background participants that we did not train. Our proposed method used projection and small-area elimination to reject this false-positive recognition. A third example for 
Table 1. Accuracy for Facial Recognition. Each video represents a different group session segment.

\begin{tabular}{|c|c|c|c|c|}
\hline Video & Duration & Person Label & Ours & Insightface \\
\hline \multirow{5}{*}{$\begin{array}{l}1 \\
\text { (Face prototypes using } \\
\text { K-means) }\end{array}$} & \multirow{5}{*}{10 seconds } & Antone39W & $36.5 \%$ & $36.5 \%$ \\
\hline & & Jaime41W & $86.7 \%$ & $84.2 \%$ \\
\hline & & Larry $40 W$ & $99.3 \%$ & $98.3 \%$ \\
\hline & & Ernesto38W & $96.5 \%$ & $95.3 \%$ \\
\hline & & Average & $79.8 \%$ & $78.6 \%$ \\
\hline \multirow{5}{*}{$\begin{array}{l}2 \\
\text { (Face prototypes using } \\
\text { Sampling) }\end{array}$} & \multirow{5}{*}{10 seconds } & Chaitanya & $95.3 \%$ & $80.3 \%$ \\
\hline & & Kenneth1P & $91 \%$ & $83.1 \%$ \\
\hline & & Jesus69P & $100 \%$ & $100 \%$ \\
\hline & & Javier67P & $100 \%$ & $69.1 \%$ \\
\hline & & Average & $96.5 \%$ & $83.1 \%$ \\
\hline \multirow{5}{*}{$\begin{array}{l}\mathbf{3} \\
\text { (Face prototypes using } \\
\text { Sampling) }\end{array}$} & \multirow{5}{*}{60 seconds } & Chaitanya & $80.0 \%$ & $56.1 \%$ \\
\hline & & Kenneth1P & $98.3 \%$ & $61.5 \%$ \\
\hline & & Jesus69P & $99.3 \%$ & $96.3 \%$ \\
\hline & & Javier67P & $80.6 \%$ & $39.0 \%$ \\
\hline & & Average & $89.5 \%$ & $63.2 \%$ \\
\hline \multirow{5}{*}{$\begin{array}{l}\mathbf{5} \\
\text { (Face prototypes using } \\
\text { Sampling) }\end{array}$} & \multirow{5}{*}{10 seconds } & Melly77W & $96.0 \%$ & $59.7 \%$ \\
\hline & & Marisol112W & $84.0 \%$ & $60.5 \%$ \\
\hline & & Cristie123W & $8.67 \%$ & $27.3 \%$ \\
\hline & & Phuong & $77.4 \%$ & $21.4 \%$ \\
\hline & & Average & $66.5 \%$ & $42.2 \%$ \\
\hline \multirow{5}{*}{$\begin{array}{l}\mathbf{5} \\
\text { (Face prototypes using } \\
\text { Sampling) }\end{array}$} & \multirow{5}{*}{60 seconds } & Alvaro70P & $96.4 \%$ & $60.8 \%$ \\
\hline & & Donna112P & $100 \%$ & $99.8 \%$ \\
\hline & & Sophial11P & $99.5 \%$ & $99.9 \%$ \\
\hline & & Average & $98.6 \%$ & $86.8 \%$ \\
\hline & & Overall Average & $86.2 \%$ & $70.8 \%$ \\
\hline
\end{tabular}

video 4 is shown in Figs. 4(c) and (f). The baseline method only succeeded in recognizing Melly77W (pink sweater) and wrongly recognized Cristie123W (lower right) as Phuong, who is actually on the far left wearing glasses. Our method used history information to address the partial occlusion issue and correctly recognized Phuong who is in the far left of Fig. 4(f). Furthermore, our method rejected the wrong assignment of Phuong because it does not allow the assignment of the same identifier to two different faces. Instead, the wrong assignment was re-assigned to Unknown. A fourth example of our method is shown in Fig. 4 ( g). In Fig. 4 $\mathrm{kg}$ ), we can see that our method works in occlusion cases. Herminio10P (dark blue polo, right) and Guillermo72P (blue T-shirt) were correctly recognized even though their faces were partial. We also present challenges in Figs. 4(h) and (i). In Fig. 4(h), Antone39W did not get recognized because 
Table 2. Recognition time for facial recognition. Each video represents a different group session segment.

\begin{tabular}{|c|c|c|c|c|c|}
\hline Video & Du & GT Faces & $\begin{array}{l}\text { Insightface } \\
\text { (seconds/frame) }\end{array}$ & $\begin{array}{l}\text { Ours } \\
\text { (seconds/frame) }\end{array}$ & $\begin{array}{l}\text { Speedup } \\
\text { factor }\end{array}$ \\
\hline 1 & 10 & 4 & 9.91 & 2.8 & $3.5 \mathrm{x}$ \\
\hline 2 & 10 & 4 & 9.96 & 0.8 & $12.5 \mathrm{x}$ \\
\hline 3 & 60 & 4 & 15.8 & 0.3 & $52.7 x$ \\
\hline 4 & 10 & 4 & 10.1 & 0.9 & $11.2 \mathrm{x}$ \\
\hline \multirow[t]{2}{*}{5} & 60 & 3 & 18.2 & 0.3 & $60.7 x$ \\
\hline & & Average & 12.8 & 1.1 & $28.1 x$ \\
\hline
\end{tabular}

he had his back facing the camera. In Fig. 4(i), Kirk28P was not recognized due to significant changes in appearance through time.

We present speed performance comparisons in Table 2 . The baseline method required 9.9 to 18.2 seconds/frame whereas our proposed method required 0.3 to 2.8 seconds/frame. On average, the proposed method was $28.1 \times$ faster. Our speedups can be attributed to our use of a reduced number of face prototypes and the fact that we do not rerun the minimum distance classifier if there is little movement in the detected faces. For example, for 3 and 4, InsightFace took a very long time (more than ten seconds/frame) because it compared each participant against (almost) ten thousand images. For 5, in addition to comparisons to about ten thousand images for the main group, InsightFace also had to compare against faces from the background groups. In comparison, our approach rejected the need to recognize background groups by applying a minimum face size constraint.

\section{Conclusion}

The paper presented a method for video face recognition that is shown to be significantly faster and more accurate than the baseline method. The method introduced: (i) clustering methods to identify image clusters for recognizing faces from different poses, (ii) robust tracking with multi-frame processing for occlusions, and (iii) multi-objective optimization to reduce recognition time.

Compared to the baseline method, the final optimized method resulted in speedy recognition times with significant improvements in face recognition accuracy. Using face prototype with sampling, the proposed method achieved an accuracy of $86.2 \%$ compared to $70.8 \%$ for the baseline system, while running 28.1 times faster than the baseline. In future work, we want to extend our approach to 150 participants in about 1,000 hours of videos. 
(a)

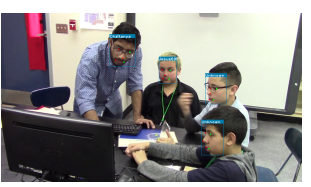

(d)

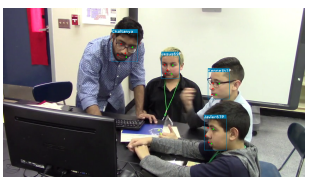

(g)

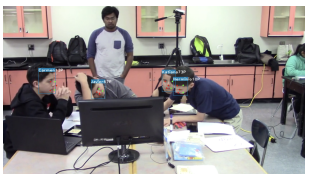

(b)
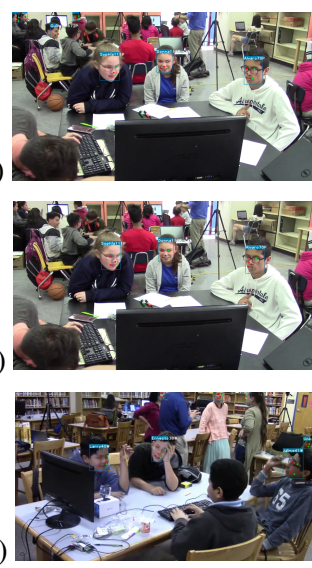

(c)
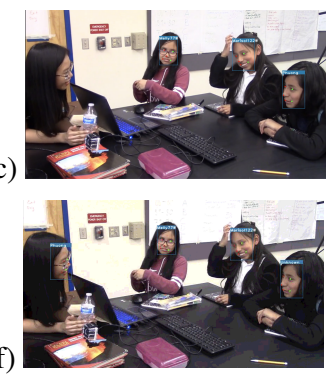

(i)

Fig. 4. Video face recognition results for three collaborative groups. The first row shows results from the use of InsightFace (baseline). The second row shows our results using the sampling method. In (g), we show successful detections despite occlusions. Results from the use of Kmeans clustering are shown in (h). Then, we show dramatic changes in appearance in (i).

\section{References}

1. Deng, J., Guo, J., Xue, N., Zafeiriou, S.: Arcface: Additive angular margin loss for deep face recognition (2019)

2. Guo, J., Deng, J., An, X., Yu, J.: Deepinsight/insightface: State-of-the-art $2 \mathrm{~d}$ and $3 \mathrm{~d}$ face analysis project (Jul 2021), https: //github.com/deepinsight/insightface

3. Huang, G.B., Ramesh, M., Berg, T., Learned-Miller, E.: Labeled faces in the wild: A database for studying face recognition in unconstrained environments. Tech. Rep. 07-49, University of Massachusetts, Amherst (October 2007)

4. Jacoby, A.R., Pattichis, M.S., Celedón-Pattichis, S., LópezLeiva, C.: Context-sensitive human activity classification in collaborative learning environments. In: 2018 IEEE Southwest Symposium on Image Analysis and Interpretation (SSIAI). pp. 1-4 (2018). https://doi.org/10.1109/SSIAI.2018.8470331

5. Learned-Miller, G.B.H.E.: Labeled faces in the wild: Updates and new reporting procedures. Tech. Rep. UM-CS-2014-003, University of Massachusetts, Amherst (May 2014)

6. Shi, W., Pattichis, M.S., Celedón-Pattichis, S., LópezLeiva, C.: Robust head detection in collaborative learning environments using am-fm representations. In: 2018 IEEE Southwest Symposium on Image Analysis and Interpretation (SSIAI). pp. 1-4 (2018). https://doi.org/10.1109/SSIAI.2018.8470355

7. Tapia, L.S., Pattichis, M.S., Celedón-Pattichis, S., Leiva, C.L.: The importance of the instantaneous phase for face detection using simple convolutional neural networks. In: IEEE Southwest Symposium on Image Analysis and Interpretation, SSIAI 2020, Albuquerque, NM, USA, March 29-31, 2020. pp. 1-4 (2020). https://doi.org/10.1109/SSIAI49293.2020.9094589

8. Tran, P.: Fast Video-based Face Recognition in Collaborative Learning Environments. Master's thesis, University of New Mexico (12 2021)

9. Zhang, K., Zhang, Z., Li, Z., Qiao, Y.: Joint face detection and alignment using multi-task cascaded convolutional networks. CoRR abs/1604.02878 (2016), http: / /arxiv.org/ $\mathrm{abs} / 1604.02878$ 